Int. J. Dev. Biol. 51: 57-66 (2007)

doi: $10.1387 / \mathrm{ijdb} .062164 \mathrm{av}$

Original Article

\title{
Stage-specific regulation of programmed cell death during oogenesis of the medfly Ceratitis capitata (Diptera, Tephritidae)
}

\author{
ATHANASSIOS D. VELENTZAS, IOANNIS P. NEZIS, DIMITRIOS J. STRAVOPODIS, ISSIDORA S. PAPASSIDERI* \\ and LUKAS H. MARGARITIS
}

Faculty of Biology, Department of Cell Biology and Biophysics, University of Athens, Athens, Greece

\begin{abstract}
In the present study, we describe novel features of programmed cell death in developing egg chambers occurring during mid- and late-oogenesis of the medfly Ceratitis capitata. During mid-oogenesis, the spontaneously degenerated egg chambers exhibit typical characteristics of apoptotic cell death. Their nurse cells contain fragmented DNA and fragmented actin, as revealed by TUNEL assay and immunolabelling, respectively. In vitro caspase activity assays and immunostaining procedures demonstrated that the atretic egg chambers acquired high levels of caspase activity. Distinct features of autophagic cell death were also observed during $C$. capitata mid-oogenesis, as revealed by the monodansylcadaverine staining approach and ultrastructural examination performed by transmission electron microscopy. Additionally, atretic egg chambers exhibit an upregulation of lysosomal proteases, as demonstrated by a procathepsin L immunolabelling procedure. At the late stages of $C$. capitata oogenesis, apoptosis and autophagy coexist, manifesting cell death features that are similar to the ones mentioned above, being also chaperoned by the involvement of an altered cytochrome conformational display. We propose that apoptosis and autophagy operate synergistically during $C$. capitata oogenesis for a more efficient elimination of the degenerated nurse cells and abnormal egg chambers.
\end{abstract}

KEY WORDS: apoptosis, autophagy, Ceratitis capitata, nurse cell, programmed cell death

\section{Introduction}

The egg chamber, or follicle, is the structural and functional unit of insect ovary. The egg chambers are formed in the anterior region of the ovary and they move towards the posterior pole as they develop during oogenesis. Thus, each ovariole contains an array of developmentally ordered egg chambers. In the meroistic polytrophic ovarioles of Drosophila melanogaster and Ceratitis capitata, each egg chamber consists of 16 germ line cells (15 nurse cells and 1 oocyte), surrounded by a monolayer of somatic epithelial cells, called follicle cells (Trougakos and Margaritis, 2002; Berg, 2005). The nurse cells support the development of the oocyte providing organelles, proteins and maternal RNAs to the oocyte through the ring canals (Mahajan-Miklos and Cooley, 1994; Trougakos and Margaritis, 2002). The development of the egg chamber has been divided into several discrete stages (fourteen according to King, 1970; twenty according to Margaritis, 1985; 1986).
Programmed cell death is an evolutionary conserved and genetically regulated process, where superfluous cells undergo self-destruction by activation of a cell suicide program (Danial and Korsmeyer, 2004). There are two major types of programmed cell death, apoptotic cell death (or type 1) and autophagic cell death (or type 2), both of which are defined by distinct morphological criteria. Apoptotic cell death is mainly characterized by nuclear condensation (pyknosis) and DNA fragmentation (karyorhexis), without major ultrastructural changes of cytoplasmic organelles. In the autophagy-mediated cell death, the major morphological feature is the accumulation of autophagic vacuoles in the cytoplasm (Bursch, 2001; 2004; Lockshin and Zakeri, 2004). Apoptosis involves the regulated activation of caspases, a family of cysteine proteases that specifically recognize and cleave critical cellular target proteins, such as nuclear lamins and other structural

Abbreviations used in this paper: FC, follicle cells; MDC, monodansylcadaverine stain; NC, nurse cell; NN, nurse cell nucleus; OC, oocyte.

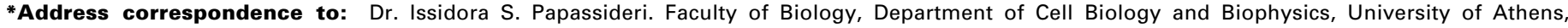
Panepistimiopolis 15784, Athens, Greece. Tel: +30-210-727-4546. Fax: ++30-210-727-4742. e-mail: ipapasid@biol.uoa.gr
} 
components, ultimately leading to cell death (Kornbluth and White, 2005). On the other hand, caspases do not seem to be required for the execution of the autophagic cell death program (Bursch, 2001; Gozuacik and Kimchi, 2004), although both types of programmed cell death have been previously reported to coexist (Clarke, 1990; Martin and Baehrecke, 2004). In both cases, the dying cells are efficiently phagocytosed by neighboring epithelial cells, or surrounding phagocytes (Krieser and White, 2002).

As it has been demonstrated before, the process of programmed cell death is activated and implemented during Diptera germarium, mid- and late-oogenesis (Drummond-Barbosa and Spradling, 2001; Nezis et al., 2000; 2001; 2002; 2003; 2005; 2006a; 2006b; 2006c, for reviews see McCall, 2004; Baum et al., 2005). Follicular atresia, a distinct pattern of developmentally regulated cell death, has been sporadically observed during Diptera mid-oogenesis (Giorgi and Deri, 1976; Nezis et al., 2000; 2003; 2006a; Uchida et al., 2004), or massively induced in response to (a) nutritional deprivation, (b) ecdysone signalling malfunction, (c) treatment with chemotherapeutic drugs (i.e. etoposide, or staurosporine) and (d) ectopic death of follicle cells in Drosophila (Buszczak et al., 1999; Chao and Nagoshi, 1999; DeLorenzo et al., 1999; Soller et al., 1999; Nezis et al., 2000; Terashima and Bownes, 2004). The atretic egg chambers contain degenerated nurse cells, mainly characterized by condensed chromatin, fragmented DNA and disorganized actin cytoskeleton network (Buszczak et al., 1999; Chao and Nagoshi, 1999; DeLorenzo et al., 1999; Soller et al., 1999; Nezis et al., 2000; 2003; 2006a). Nurse and follicle cell death programs are also required for the normal maturation of the developing follicles during the late stages of Drosophila, Bactrocera oleae and C. capitata oogenesis (Cavaliere et al., 1998; Foley and Cooley, 1998; Nezis et al., 2000; 2001; 2002; 2003; 2006b; 2006c, for reviews see McCall, 2004; Baum et al., 2005).

In the present study, we demonstrate, for the first time, that apoptosis and autophagy function together during mid- and lateoogenesis of the medfly $C$. capitata. Apoptosis in mid-oogenesis exhibits caspase activation that is tightly associated with DNA fragmentation and actin cytoskeleton disorganization. Interestingly, this apoptotic process seems to be implemented through a cytochrome c-independent mechanism. On the other hand, autophagy is mainly characterized by the formation of autophagosomes and the production of cathepsin-like proteases that are likely related to the fragmentation of the yolk spheres. During late-oogenesis, apoptosis is executed through a limited caspase activation program, chaperoned with a modified cytochrome c conformational display. At the end of apoptosis, autophagy strongly facilitates the more efficient clearance of the nurse cell remnants by the adjacent follicle cells.

\section{Results}

The nurse cells of the atretic egg chambers evince both apoptotic and autophagic cell death features during $C$. capitata mid-oogenesis checkpoint.

The development of the egg chamber of the medfly C. capitata is divided into 14 distinct stages (Mouzaki and Margaritis, 1991; Nezis et al., 2003; Trougakos and Margaritis, 2002). In the present study, we thoroughly examined the developmental stages
7-13 (Fig. 1). We have previously shown that egg chambers during $C$. capitata mid-oogenesis degenerate in low frequency. These sporadically produced egg chambers are characterized by abnormal morphology and loss of their cellular compartmentalized organization and integrity compared to the normal ones of the same developmental stages (Nezis et al., 2003). We have further analyzed these abnormal egg chambers and found that their nurse cells contain condensed chromatin and fragmented DNA (Figs. 2 A-C). In order to unveil the alterations of actin cytoskeleton network, we applied an immunolabelling approach using the anti-fractin antibody as a specific probe. During apoptosis, the beta actin cytoskeletal component is specifically cleaved by activated caspases. This cleavage results in the release of approximately 130 amino acid residues $(\sim 15 \mathrm{kDa})$ from the carboxyl-terminus of the beta actin subunit, generating a truncated $32 \mathrm{kDa}$ amino-terminal fragment of actin, widely known as fractin (Yang et al., 1998; Rossiter et al., 2000). All the abnormal egg chambers tested are characterized by intense anti-fractin immunostaining signals mainly in the nurse cell cluster, directly indicating the generation of the $32 \mathrm{kDa}$ truncated actin fragment (Fig. 2D and data not shown).

Using the rhodamine-conjugated substrate of active caspases Red-VAD-FMK, we clearly demonstrate the occurrence of highly activated caspases in all the atretic egg chambers tested (Fig. $2 \mathrm{E})$. Moreover, through an immunolabelling approach, using the CM1 antibody that specifically recognizes an activated form of the Drosophila caspase Drice (Laundrie et al., 2003; Peterson et al., 2003), we detect positive staining signals in the degenerating nurse cell cluster, indicating the presence of activated caspase3-like (Drice, or Drice-like) proteases (Fig. 2 F,G). On the contrary, no positive signal for activated caspases could ever be observed

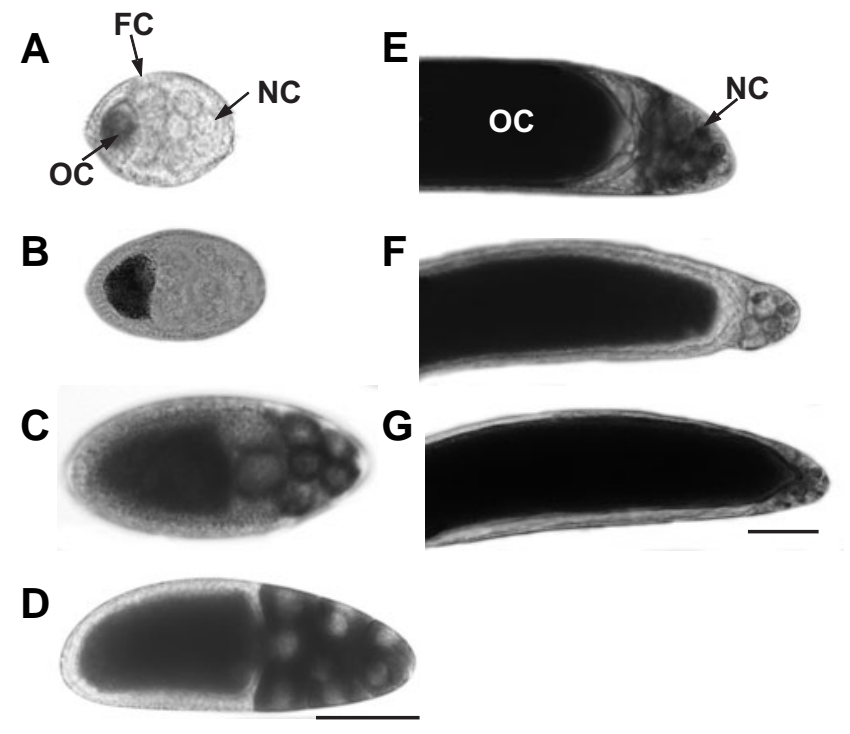

Fig. 1. The development of the egg chamber during oogenesis of the medfly $\boldsymbol{C}$. capitata. The general morphology of representative egg chambers at the developmental stages 7-13 is illustrated in light micrographs (A-G). The size of the egg chamber increases gradually up to the late stages of oogenesis. (A) stage 7, (B) stage 8, (C) stage 9, (D) stage 10, (E) stage 11, (F) stage 12 and (G) stage 13. FC, follicle cells; NC, nurse cells and OC, oocyte. Scale bars: (A-D) $50 \mu \mathrm{m}$; (E-G) $100 \mu \mathrm{m}$. 

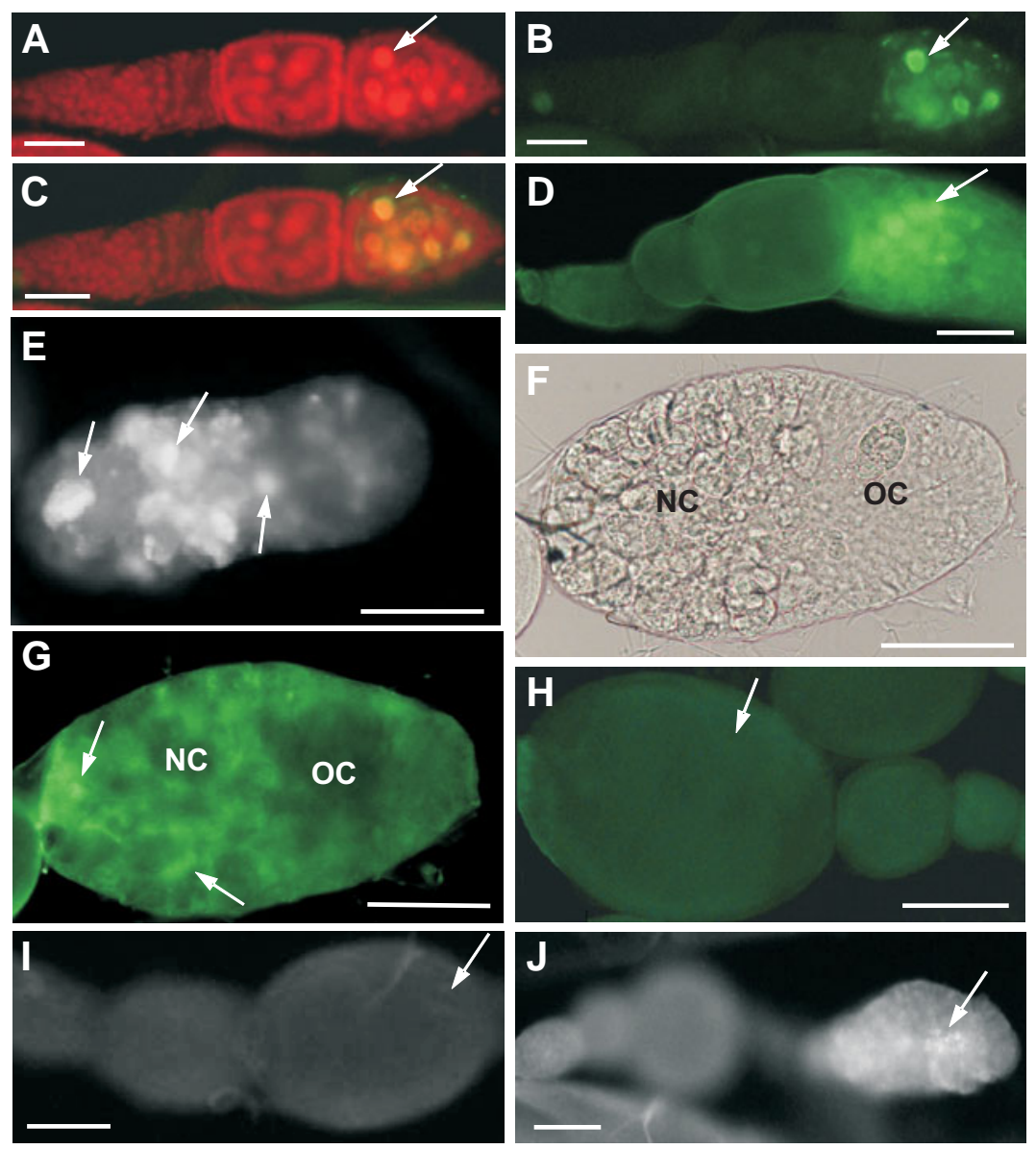

Fig. 2. Spontaneously induced programmed cell death during $C$. capitata mid-oogenesis exhibits typical apoptotic features, such as DNA fragmentation, formation of a truncated beta actin fragment and activation of a caspase repertoire program. (A-C) Fluorescent micrographs of a representative C. capitata atretic egg chamber during mid-oogenesis after propidium iodide staining (A) and TUNEL assay (B). (C) Merge of the $(A)$ and (B) images. Intense signals (respective arrows) are clearly observed in the nurse cell nuclei (NN) of stage 7-8 egg chambers and not in previous developmental stages. (D) Fluorescent micrograph of an ovariole after immunostaining with the antifractin antibody. The intense positive signals observed in the nurse cells (arrow) of stage 7-8 egg chamber demonstrate the presence of fragmented actin. Note the absence of detectable signal in the previous developmental stages. (E) Red-VAD-FMK strong staining of stage 7-8 atretic egg chambers reveals the presence of active caspases in the degenerating nurse cell cluster (arrows). (F-H) Immunostaining with the CM1 antibody evinces the activation of the caspase family member Drice (or Drice-like proteins) in the nurse cells of a representative stage 7-8 atretic egg chamber (G, arrows). (F) Phase contrast light micrograph of (G). (H) No staining can be observed in a normal egg chamber of the same stage (arrow). (I) Fluorescent micrograph after an immunolabelling approach using the 2aG8 anti-cytochrome $c$ monoclonal antibody. No traceable staining could ever be observed in all the tested atretic egg chambers (arrow). (J) Fluorescent micrograph after immunostaining reactions using the anti-Dronc antibody. The stage 7-8 atretic egg chamber exhibits positive signal (arrow). NC, nurse cells and OC, oocyte. Scale bars: $50 \mu \mathrm{m}$. in the normal egg chambers during mid-oogenesis (Fig. $2 \mathrm{H}$ ). Additionally, immunostaining reactions using the anti-Dronc polyclonal antibody demonstrates a stage-specific (7-8) accumulation of the Dronc-like caspase in the nurse cell cluster of the atretic egg chambers (Fig. 2J). In order to identify whether cytochrome $\mathrm{c}$ plays any role in the observed caspase activation program, we applied a specific immunostaining approach using the monoclonal antibody 2aG8 (Mueller and Jemmerson, 1996). This particular antibody has been previously shown to recognize an altered and exposed epitope of cytochrome c, which is specifically unmasked during programmed cell death in Drosophila melanogasteregg chambers (Varkey et al., 1999). As illustrated

Fig. 3. Programmed cell death during $C$. capitata mid-oogenesis manifests typical features of autophagy. (A) Monodansylcadaverine (MDC) staining of an atretic stage 7-8 egg chamber. The positive strong signals reflect the presence of autophagic vacuoles (arrows). (B) A normally developed stage 7-8 egg chamber (arrow), with a prominent absence of autophagosome formation (MDC negative staining). (C) Immunostaining with an anti-procathepsin L specific antibody, revealing a strong and punctate staining pattern in the abnormal egg chamber during mid-oogenesis (arrows). (D) Transmission electron micrograph of an atretic stage 7-8 egg chamber, illustrating the presence of degenerated yolk spheres (arrows). (E) Transmission electron micrograph of an abnormal stage 7-8 egg chamber demonstrating the occurrence of large autophagosomes (arrows). They contain numerous vesicles of different sizes and multi-lamellar membranes. Scale bars: $(A-C) 50 \mu \mathrm{m}$; (D) $1 \mu \mathrm{m}$; (E) $10 \mu \mathrm{m}$. in Fig. 2l, we were not able to obtain any detectable anticytochrome $\mathrm{c}$ immunoreaction signal in any of the atretic egg chambers tested $(n=250)$, suggesting the absence of the cytochrome c involvement (or at least the display, or unmasking of the respective epitope) in the execution of the nurse cell cluster apoptotic death during mid-oogenesis.

To answer the question whether autophagy occurs during mid-
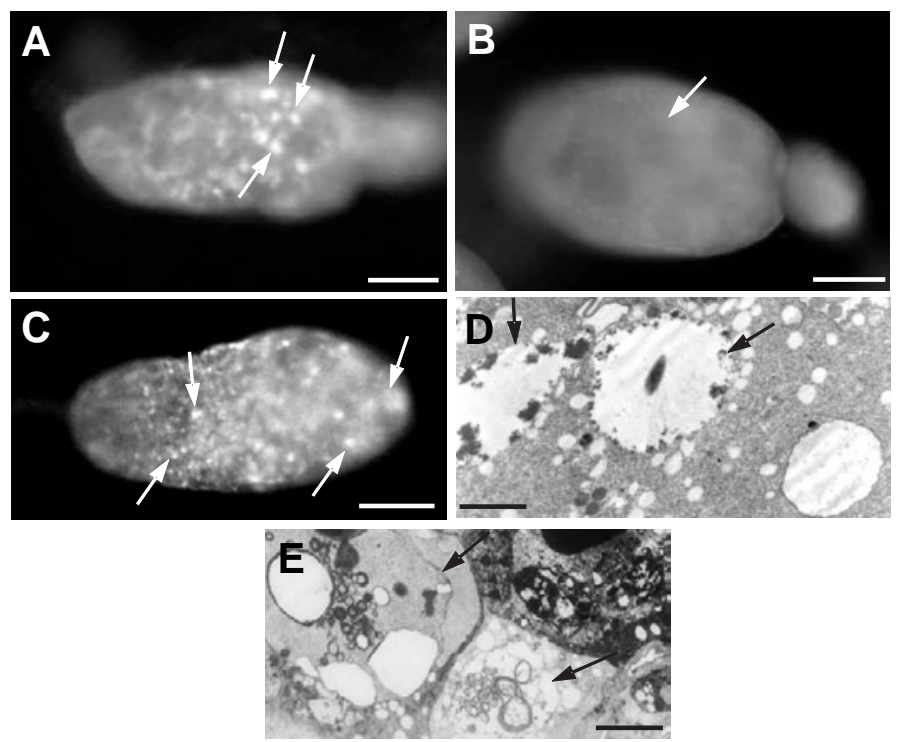
oogenesis of $C$. capitata, we first used monodansylcadaverine staining for tracing the autophagic vacuoles. Several positive signals are clearly observed in the nurse cell cluster of all the abnormal egg chambers tested (Fig. 3A), while the physiologically developed ones are completely devoid of any detectable staining (Fig. 3B). Interestingly, an immunostaining approach using the anti-procathepsin $L$ polyclonal antibody, raised against the Sarcophaga peregrine protein family member (Uchida et al., 2001), clearly reveals an intense and punctate staining pattern all over the non-compartmentalized atretic egg chamber (Fig. 3C).

The examination of the ultrastructural morphology of the sporadically produced atretic egg chambers discloses the occurrence of several degenerated yolk spheres with fragmented and degraded contents (Fig. 3D). Moreover, the atretic egg chambers are characterized by the presence of large autophagosomes that are scattered in all the areas of the abnormal egg chamber, containing numerous vesicles and multi-lamellar membranes of various sizes (Fig. 3E and data not shown).

Ovarian nurse cells of Ceratitis capitata during late-oogenesis exhibit both apoptotic and autophagic cell death

It was previously shown that the nurse cells of $C$. capitata egg chambers normally degenerate during the late stages of oogenesis (Nezis et al., 2003). Using the acridine orange staining, we demonstrate that during the developmental stages 12 and 13 all
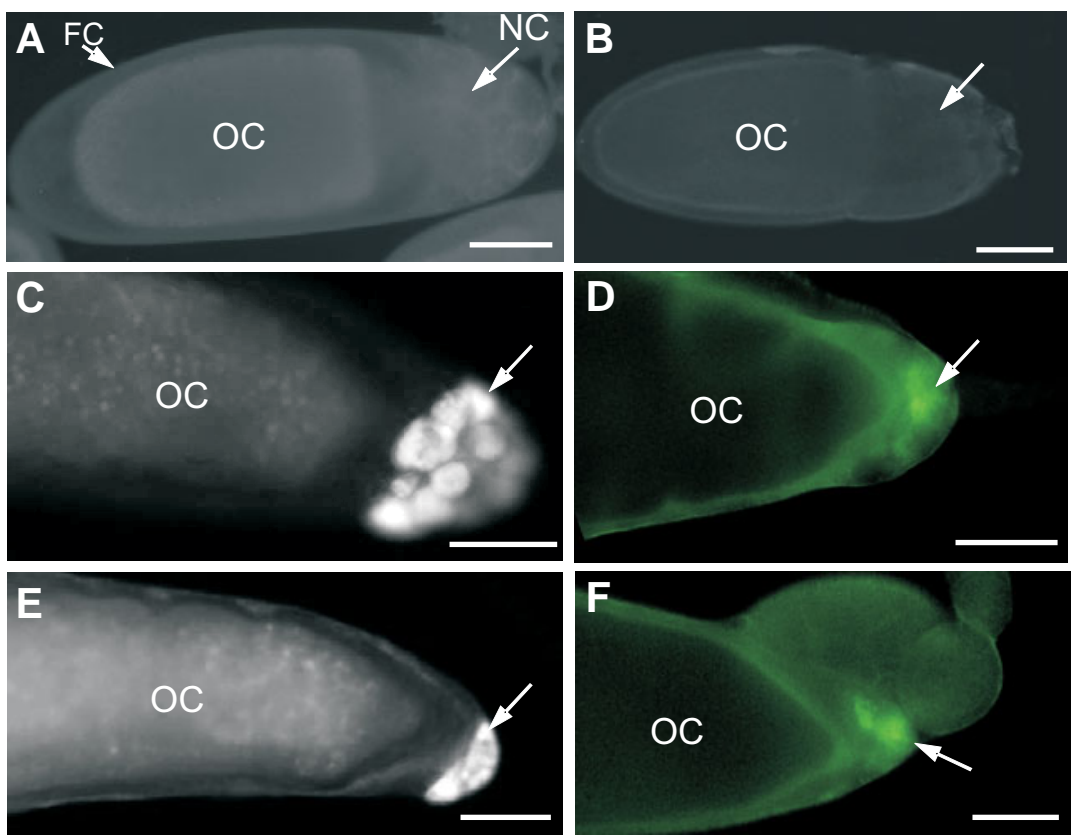

Fig. 4. Programmed cell death during $C$. capitata late-oogenesis exhibits typical apoptotic features, such as DNA fragmentation and fragmented actin. (A,C,E) Stage-specific DNA fragmentation, as revealed by an acridine orange staining approach. (B,D,F) Developmentally regulated fractin production, as it is disclosed by an anti-fractin immunostaining. (A,B) Stage 11 egg chambers. Observe the complete absence of traceable positive signals for both acridine orange staining (A) and anti-fractin immunolabelling (B), all over the egg chamber. (C,D) Stage 12 egg chambers, exhibiting intense positive signals exclusively produced in the nurse cell cluster after both acridine orange staining (C) and anti-fractin immunolabelling (D) (respective arrows). (E,F) During stage 13, strong positive signals for both applied staining approaches can be observed, respectively (arrows). NC, nurse cells; FC, follicle cells; OC, oocyte. Scale bars: $100 \mu \mathrm{m}$. the nurse cell nuclei become apoptotic and contain fragmented DNA (Fig. 4 C,E), while no DNA fragmentation event can be mers, using an antibody staining approach for frag. On the contrary, fragmented actin immunostaining urse cell cluster (Fig. 4 D,F).

To elucidate the potential implication of cytochrome $\mathrm{c}$ in the 2aG8 (Mueller and Jemmerson, 1996) at the developing $C$. capitata egg chambers. No staining was revealed in all three partments of a representative stage 10 egg chamber (Fig. unostaining, which is mainly dispersed in all the respective the next devis. specks are clearly observed in the nurse cell compartment (Fig. $5 G$ ). Thus, it is very likely that cytochrome c constitutes an essential signalling component for the effective execution of the C. capitata nurse cells apoptotic program.

A similar staining pattern can be disclosed during C. capitata late-oogenesis, using the anti-Dronc polyclonal antibody. A diffused anti-Dronc immunostaining signal is primarily detected during stage 11 mainly in the respective cytoplasmic areas of the nurse cell compartment (Fig. 5D). This dispersed staining profile remains unaffected during the next developmental stage 12 (Fig. 5F), while the stage 13 is mainly characterized by a more focalized signal (Fig. $5 \mathrm{H}$ ). On the other hand, all stage $10 \mathrm{egg}$ chambers are completely devoid of any immunostaining reaction in all three compartments (oocyte, nurse cells and follicle cells) (Fig. 5B).

In order to illuminate the actual participation and contribution of active caspases in the cell death program of $C$. capitata nurse cells, we applied an active caspase in situ assay, using the Red-VADFMK fluorescent inhibitor as the specific detecting probe. As it is clearly illustrated in Fig. 6A, no positive signals for active caspases could ever be detected in any area of the stage $10 \mathrm{egg}$ chambers. Similar negative results were also obtained using the CM1 polyclonal antibody that specifically recognizes an activated form of the Drosophila caspase family member Drice (Peterson et al., 2003) (Fig. 6B). During the next developmental stage 11, Red-VADFMK positive signals for active caspases are clearly detected in the nurse cell cluster, especially in the nurse cells adjacent to the oocyte (Fig. 6C), while no traceable CM1 immunostaining reaction is observed in any of the egg chamber compartments (Fig. 6D). In contrast, the following stage 12 is mainly characterized by distinct signals produced after the applica- 

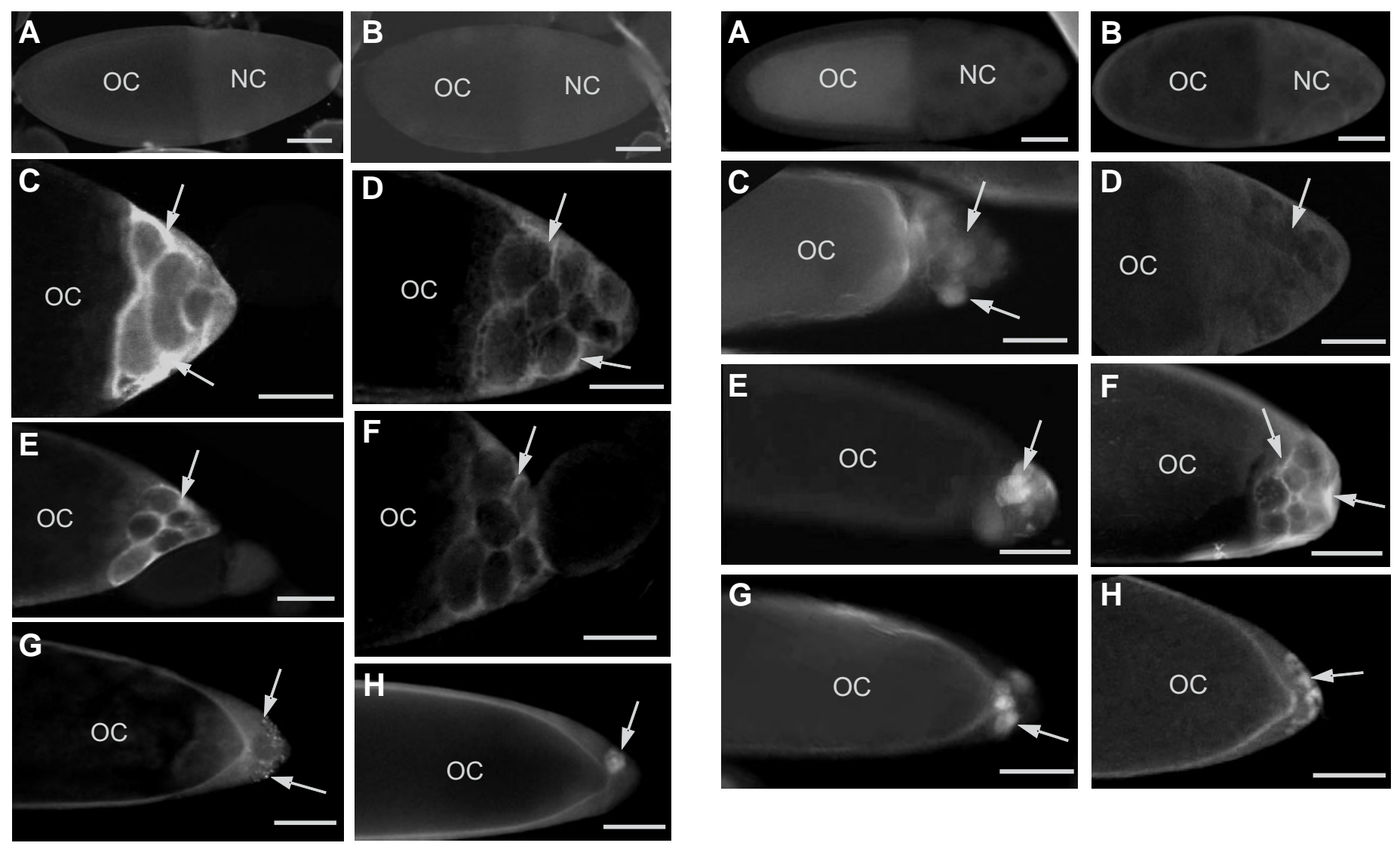

Fig. 5 (Left Column). Late stage egg chambers of $C$. capitata exhibit the presence of an altered cytochrome conformational display and upregulation of Dronc-like proteins. Confocal and conventional light micrographs after immunostaining of developing egg chambers with the $2 a G 8$ anti-cytochrome c monoclonal antibody (A, C, E, G) and an anti-Dronc antibody (B, D, F, H). (A,B) Stage 10 egg chambers, showing no detectable staining for cytochrome $c$ and Dronc, respectively. (C,D) During stage 11, intense diffused staining can be clearly observed in the nurse cell cytoplasm for both cytochrome $c$ and Dronc-like proteins, respectively (arrows). A similar pattern is also obtained during the following stage 12 (E,F), (see respective arrows). During stage 13, the obtained immunostaining respective pattern for either cytochrome c or Dronc-like caspases becomes more focalized (G,H), (see arrows). The staining observed at the oocyte periphery is due to autofluorescence of chorion. NC, nurse cells and OC, oocyte. Scale bars: $100 \mu \mathrm{m}$.

Fig. 6 (Right Column). Limited caspase activation occurs in a stage-specific manner during $\boldsymbol{C}$. capitata late-oogenesis. Confocal and conventional light micrographs of representative egg chambers after Red-VAD-FMK staining $(\mathbf{A}, \mathbf{C}, \mathbf{E}, \mathbf{G})$ and an immunolabelling approach using the CM1 antibody (B, D, F, H). (A,B) Late stage 10 representative egg chambers showing the complete absence of caspase activity for both applied staining approaches. (C,D) Positive signals for the Red-VAD-FMK staining reagent are detected in the nurse cell cluster during stage 11 (C), while no traceable staining can be observed for an activated Drice (or Drice-like) caspase (D) (arrows). (E-H) Nurse cell compartment-specific staining reactions for both Red-VAD-FMK (E,G) and CM1 (F,H) labelling approaches are revealed during stage $12(E, F)$ and stage $13(G, H)$ of C. capitata oogenesis (arrows). NC, nurse cells and OC, oocyte. Scale bars: $100 \mu \mathrm{m}$.

tion of either the Red-VAD-FMK or CM1 labelling approach, as it is demonstrated in Fig. $6 \mathrm{E}, \mathrm{F}$, respectively. During the developmental stage 13, the obtained, from both techniques, positive reactions are focalized in size, producing a more distinct and punctate staining pattern (Fig. $6 \mathrm{G}, \mathrm{H}$ ).

In order to examine whether an autophagic cell death process is essentially involved during $C$. capitatalate-oogenesis, we used monodansylcadaverine staining for tracing the occurrence of autophagic vacuoles. Stage 10 and 11 egg chambers do not appear to develop any positive staining signal (Fig. 7A and data not shown). On the contrary, during the following stages 12 and 13 punctate discrete monodansylcadaverine staining patterns are distinguished in the nurse cells of the developing egg cham- bers (Fig. $7 \mathrm{C}, \mathrm{E}$ ). When the ultrastructural organization of the stage 12 and 13 egg chambers was examined, several autophagosomes, containing numerous vesicles, membranes and dense masses, are readily observed in the degenerated nurse cells (Fig. $7 \mathrm{G}, \mathrm{H}$ and data not shown). These autophagosomes are mainly located in close proximity to the actin bundles (Fig. 7G).

In addition, the generation of autophagic vacuoles is escorted by a distinct immunostaining profile obtained through the usage of the anti-procathepsin $\mathrm{L}$ polyclonal antibody that has been raised against the Sarcophaga peregrineprotein family member (Uchida et al., 2001). As it is illustrated in Fig. 7D, a representative stage 12 egg chamber is mainly characterized by punctate staining 

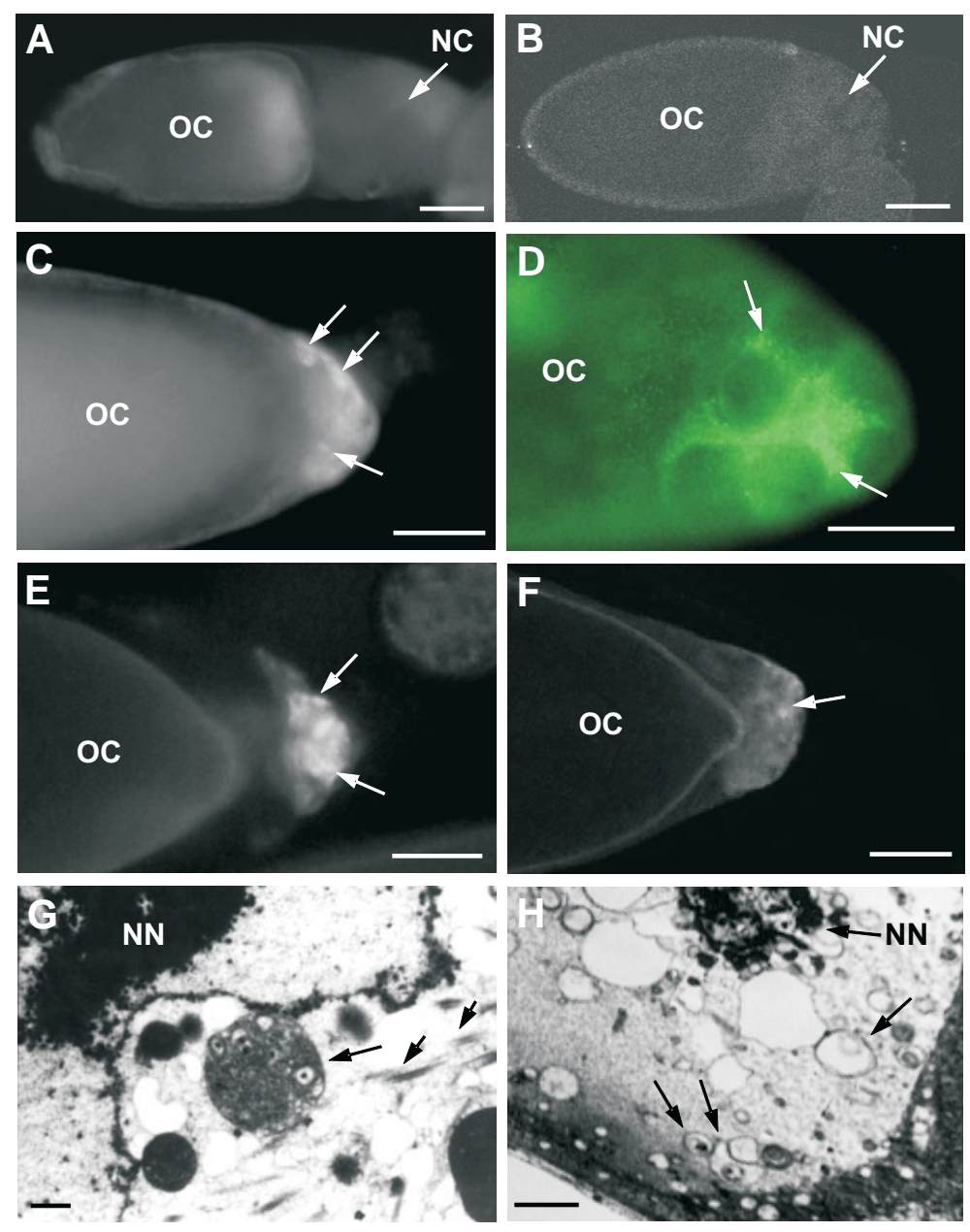

Fig. 7. Programmed cell death during $C$. capitata late-oogenesis is characterized by typical features of autophagy and upregulation of lysosomal enzymes. (A,C,E) Monodansylcadaverine $(M D C)$ staining and (B,D,F) antiprocathepsin L immunolabelling. (A,B) During stage 11, no traceable positive signal can be observed after either MDC staining $(A)$, or anti-procathepsin $L$ immunolabelling (B). (C-F) During the following developmental stages 12 (C, D) and $13(\mathbf{E}, \mathbf{F})$, distinct strong $M D C(C, E)$ and anti-procathepsin $L(D, F)$ staining patterns are clearly distinguished in the degenerated nurse cell cluster (respective arrows). (G) Transmission electron micrograph illustrating the occurrence of an autophagosome (arrow) in a nurse cell during stage 12. The particular autophagosome is located in close proximity to actin bundles (small arrows). (H) Transmission electron micrograph demonstrating the occurrence of several autophagosomes (arrows) in a nurse cell during stage 13 (NN-arrow: nurse cell nuclear remnants). NC, nurse cells; NN, nurse cell nuclei and OC, oocyte. Scale bars: (A-F) $100 \mu \mathrm{m}$; (G) $1 \mu \mathrm{m}$; (H) $5 \mu \mathrm{m}$.

restricted to the nurse cell compartment. During the following stage 13 , the nurse cells seem to contain intense anti-procathepsin $\mathrm{L}$ immunostaining signals (Fig. $7 \mathrm{~F}$ ). On the other hand, all the analyzed stage 11 egg chambers are devoid of any detectable staining in all three compartments (Fig. 7B).

\section{Discussion}

Programmed cell death occurs sporadically during mid-oogenesis and normally during late-oogenesis and results in the degeneration of the nurse cell cluster in Ceratitis capitata (Nezis et al.,
2003). The efficient execution of this cell death program involves chromatin condensation, DNA fragmentation and phagocytosis of the cellular debris by adjacent follicle cells (Nezis et al., 2003). In the present study, we reveal for the first time that apoptotic and autophagic cell death coexist during the above developmental stages.

During mid-oogenesis, the nurse cells of the spontaneously degenerated egg chambers contain fragmented DNA and fragmented beta actin, as well. The pancaspase VADFMK substrate assay clearly reveals that the caspase repertoire program is highly activated at these atretic egg chambers. Moreover, a distinct caspase-3-like (Drice, or Drice-like) protein is also activated, as it is demonstrated by the CM1 immunolabelling approach. Recent studies in Drosophila have shown that caspases and their inhibitors play a significant role in the execution of the mid-oogenesis cell death. Over-expression of an active form of the effector caspase Dcp-1 in the ovary results in the degeneration of egg chambers during mid-oogenesis (Peterson etal., 2003). This phenotype is also characterized by high levels of the active caspase Drice and can be suppressed by overexpression of the apoptotic inhibitor DIAP-1 (Peterson et al., 2003). Additionally, flies defective for Dcp-1 display a lack of sporadically degenerated stage 7-8 egg chambers (Laundrie et al., 2003; Nezis et al., 2005). Thus, it is reasonable to assume that the $C$. capitata homologous respective members of Dcp-1 and Drice caspases are responsible for the execution of the sporadically observed cell death program during mid-oogenesis. Interestingly, homologous caspase family members have been shown to exist in other Diptera species, like the mosquito Anopheles stephensi (Abraham et al., 2004). The regulation of midoogenesis cell death in Drosophila is mainly controlled by the proper balance between juvenile hormone and ecdysone (Soller et al., 1999; Buszczak et al., 1999). Interestingly, the $C$. capitatahomologous member of the ecdysone receptor, $\mathrm{CcEcR}$, is expressed at its highest levels during mid-oogenesis (Verras et al., 2002). Additionally, in mosquitoes there is a significant increase in the levels of ecdysone following a blood meal, which allows for the onset of vitellogenesis (Raikhel et al., 2002). Thus, it is very likely that ecdysone and juvenile hormone levels can also control whether an egg chamber survives or perishes during $C$. capitata mid-oogenesis. Recent studies in Drosophila revealed that the gene for Dronc caspase is significantly upregulated after 20E-hydroxy-ecdysone treatment, or poor nutrition that results in degeneration of the egg chambers during mid-oogenesis (Terashima and Bownes, 2005). The upregulation and accumulation of the Dronc-like protein we observed herein is in absolute agreement with the above results.

In atretic follicles of the mosquito Culex pipiens pallens, ovarian cathepsin-like proteinases, which have been accumulated within the developing oocyte primarily for embryogenesis, are activated to digest yolk proteins and other cellular substrate structures (Uchida et al., 2001). Additionally, the atretic follicles of the olive fruit fly Bactrocera oleaecontain several fragmented yolk spheres (Nezis et al., 2006a). The above observation is very similar to the one we describe herein in degenerating egg chambers of $C$. capitata. In the present study, anti-procathepsin L 
immunolabelling shows intense punctate staining in the atretic egg chambers. We could speculate that ovarian cathepsin-like proteinases are specifically activated in $C$. capitataabnormal egg chambers in order to digest yolk proteins and other cellular substrate structures. It has been also previously reported that cathepsins are implicated in the activation of caspases (Bursch, 2001; Chwieralski et al., 2006) Thus, it is likely that the degeneration of the egg chambers during mid-oogenesis is also regulated by a stage-specific activation of distinct endogenous ovarian proteinases.

Autophagy is defined as the process by which a portion of the cytosol and organelles are sequestered in a random manner by isolation membranes. Such an isolation membrane engulfs portions of the cytoplasm and forms a double-membrane-layered initial autophagic vacuole, or autophagosome, that fuses with a lysosome and generates the autolysosome, where the cellular components of the vacuole are degraded (Bursch, 2001; 2004; Lockshin and Zakeri, 2004; Muller et al., 2004; Juhasz and Sass, 2005). In multicellular organisms, autophagy was integrated to cell death events in post-mitotic cells in order to cope with the mass of cytoplasm needed to be degraded in a short period of time, thereby lowering the exigency for macrophages, or epithelial cells, to clear up the corpses (Lockshin and Zakeri, 2004). During mid-oogenesis of $C$. capitata, the sporadically degenerated egg chambers contain large autophagosomes, demonstrating the stage-specific activation of a distinct autophagic cell death program. It is very likely that the cellular contents of the nurse cells and oocyte compartment of the atretic egg chambers are partially degraded and lysed inside the generated autophagosomes. Thus, the follicle cells that are committed to phagocytose and wipe up the nurse cell and oocyte remnants (Nezis et al., 2003) have less work to fulfill. We propose that an autophagic cell death program strongly supports the follicular epithelium phagocytotic activities for a rapid and more efficient clearance of the cellular debris in the atretic egg chambers during $C$. capitatamid-oogenesis.

During the late stages of oogenesis the nurse cells exhibit fragmented DNA and actin, an altered cytochrome c conformational display (differential epitope unmasking) and a caspase repertoire activation program. We have previously demonstrated that the actin cytoskeleton network is reorganized during lateoogenesis in C. capitata (Nezis et al., 2003), commencing by developmental stage 11 when actin bundles are formed. The formation of these bundles is unlikely associated with a caspasemediated actin cleavage, since at this particular stage (11) no staining for fragmented actin can be detected, in all the tested egg chambers. The autophagosomes observed in the degenerating nurse cells during late-oogenesis are mainly located in close proximity to actin bundles. Actin cytoskeleton integrity has been previously shown to play an essential role in the execution of autophagic cell death, while the sequestration of cytoplasmic structural components involves the action of intermediate filaments, as well (Bursch et al., 2000; Bursch, 2004). Thus, we suggest that the caspase-independent actin bundles formation is necessary for the implementation of autophagic cell death during C. capitata late-oogenesis.

In Drosophila egg chambers, the anti-cytochrome c monoclonal antibody $2 \mathrm{aG} 8$ has been proposed to recognize an altered configuration of the protein and specifically labels dying cells
(Varkey et al., 1999). Through utilization of the particular antibody, we were able to reveal an altered cytochrome c conformational display that was initially detected in the nurse cells of stage 11 egg chambers, exhibiting a rather diffuse cytoplasmic staining. In the same stage egg chambers, we observed an upregulation of Dronc (or Dronc-like) caspase family member that is tightly associated with the activation of caspase repertoire program, as this is clearly revealed by the VAD-FMK fluorescent inhibitor assay. We propose that during the developmental stages 11 and 12 cytochrome c likely activates the Dronc (or Dronc-like) caspase family member, which, in turn, is able to trigger the Drice (or Dricelike) proteolytic activities that are likely associated with the efficient implementation of the $C$. capitata nurse cell death program. Caspase activation during late-oogenesis in $C$. capitata seems to occur in limited levels, compared to mid-oogenesis since the level and localization of activity differ among the various developmental stages. This finding completely corroborates previously reported results in Drosophila (Peterson et al., 2003), suggesting that the careful and limited caspase activation in dying nurse cells may prevent the destruction of the connected oocyte.

Interestingly, in contrast to what has been observed during late-oogenesis, the mid-oogenesis nurse cells apoptosis seems to be a cytochrome c-independent process, even though an alternative implication of the cytochrome $\mathrm{c}$ signalling component can not be excluded. This differential contribution of cytochrome $c$ in the execution of mid- and late-oogenesis apoptotic programs comprises a novel and important issue that needs further exploration and enlightenment.

Distinct features of a nurse cell autophagic death can be detected during the late developmental stages of $C$. capitata oogenesis, as it is clearly evidenced by the presence of several autophagosomes and the accumulation of the procathepsin $L$ family member. It has been previously reported that cathepsinlike proteinases are implicated in the activation of the caspase repertoire program (Bursch, 2001; Chwieralski et al., 2006). The contribution and critical role of lysosomal enzymes in the execution of mid- and late-oogenesis respective apoptotic programs comprise novel interesting issues that await further exploration.

In conclusion, our data contribute to the elucidation of the mechanisms of programmed cell death during oogenesis in the medfly $C$. capitata. We propose that during mid-oogenesis the cell death program, specifically implemented in atretic egg chambers, causes accumulation and activation of Dronc, or Dronc-like proteins independently from cytochrome $c$ involvement. Drice, or Drice-like proteins are subsequently activated, likely inducing DNA fragmentation events and fractin generation that is tightly associated with the actin cytoskeleton disorganization. In parallel, several autophagosomes are formed in order to lower the exigency of the adjacent follicle cells to clear up the nurse cell corpses. During late-oogenesis, the cell death stimuli cause an alteration in the cytochrome c conformational display that is likely implicated in the Dronc, or Dronc-like caspase activation. The subsequently activated Drice, or Dricelike caspases cause DNA fragmentation and alterations in the actin cytoskeleton network. In parallel, autophagosomes are formed in order to efficiently support the adjacent follicle cells to absorb the nurse cell corpses. We propose that after completion of the execution phase of apoptosis, autophagy helps for 
the clearance of the apoptotic corpses from the adjacent follicular epithelium.

\section{Materials and Methods}

\section{Ceratitis capitata culturing}

C. capitata (Diptera, Tephritidae) adult insects were kept in a $25^{\circ} \mathrm{C}$ culture room, fed on standard diet (10\% yeast, $40 \%$ sugar in water) and slightly etherized before dissection. Dissections were carried out in cold Ringer's solution and ovaries were separated into single ovarioles. Staging of the developing egg chambers during oogenesis was accomplished according to Margaritis (1986), Mouzaki and Margaritis (1991) and Nezis et al. (2001; 2003).

\section{Acridine orange staining}

Ovaries were dissected in Ringer's solution and separated into individual egg chambers. The egg chambers were then incubated with $1.6 \mu \mathrm{M}$ acridine orange in Ringer's solution for $5 \mathrm{~min}$ in the dark. Subsequently, they were washed for 5 min in Ringer's solution and immediately mounted onto vidal glass slides in fresh Ringer's solution. Elapsed time from dissection to the end of the viewing was restricted to $20 \mathrm{~min}$. Samples were examined using a Nikon Eclipse TE 2000-S fluorescent microscope.

\section{Double staining with propidium iodide and TUNEL assay}

Egg chambers were fixed in PBS containing $4 \%$ formaldehyde plus $0.1 \%$ Triton X-100 for $25 \mathrm{~min}$. After three washes in PBS for 5 min each, the egg chambers were incubated with PBS containing $20 \mu \mathrm{g} / \mathrm{ml}$ proteinase $\mathrm{K}$ for $15 \mathrm{~min}$. Then, they were washed three times in PBS for $5 \mathrm{~min}$ each and incubated with the TUNEL reaction mixture for 3 hours at $37^{\circ} \mathrm{C}$ in the dark. Next, the egg chambers were washed three times in PBS for $5 \mathrm{~min}$ each, subsequently incubated with PBS containing $800 \mu \mathrm{g} / \mathrm{ml}$ RNaseA for $60 \mathrm{~min}$ and finally stained with $700 \mathrm{ng} / \mathrm{ml}$ propidium iodide in PBS for $15 \mathrm{~min}$. At the end of the procedure, the egg chambers were washed three times in PBS, mounted in anti-fading mounting medium and viewed using a Nikon Eclipse TE 2000-S fluorescent microscope.

\section{Active caspase in situ assay}

Ovaries were dissected in Ringer's solution and the separated individual egg chambers were examined for the presence of active caspases using the Red-VAD-FMK detection kit (rhodamine-conjugated inhibitor of active caspases) (Oncogene Research Products, San Diego, USA). After the egg chambers were briefly rinsed with PBS, they were immediately transferred into the reaction mixture and incubated for $30 \mathrm{~min}$ at $25^{\circ} \mathrm{C}$ in the dark. The treated egg chambers were subsequently rinsed three times with wash buffer, washed twice with PBS and finally put on a glass slide. The specimens were immediately observed under a Nikon Eclipse TE 2000-S fluorescent microscope using the appropriate filters.

\section{Immunohistochemical procedures}

Ovaries were dissected in Ringer's solution and separated into individual egg chambers. The egg chambers were fixed in PBS containing $4 \%$ formaldehyde for $20 \mathrm{~min}$. After fixation, the samples were washed with PBS and subsequently incubated in PBS containing $0.1 \%$ Triton X-100 for $10 \mathrm{~min}$. After the egg chambers were extensively washed with PBS, they were incubated in PBS containing $20 \mathrm{mM}$ glycine and 1.5\% BSA for 45 min. The primary antibodies used in the present study are given and commented as following: (a) the anti-fractin polyclonal antibody (Chemicon International, USA) was utilized at a dilution of 1:1000, (b) the mouse anticytochrome c monoclonal antibody of the 2aG8 clone was kindly obtained by Professor Ron Jemmerson (University of Minnesota, USA) and used at a concentration of $22 \mu \mathrm{g} / \mathrm{ml}$, (c) the rabbit CM1 polyclonal antibody was supplied by BD Biosciences Pharmingen (USA) and used at a dilution of $1: 1000$, (d) the rabbit anti-Dronc polyclonal antibody that recognizes both the precursor $(50 \mathrm{kDa})$ and the processed $(39 \mathrm{kDa})$ form of the Drosophila caspase family member Dronc (Waldhuber et al., 2005) was provided by
Professor Claudia Petritsch (Ludwig-Maximilian-University Munich, Germany) and utilized at a dilution of 1:200 and (e) the rabbit anti-procathepsin L (Sarcophaga peregrine) polyclonal antibody was supplied by Professor Koichi Homma (Faculty of Pharmaceutical Sciences, University of Tokyo, Japan) and used at a final dilution of 1:1000. After incubation with the respective primary antibody, the treated egg chambers were washed four times with PBS-T (PBS containing $0.1 \%$ Triton X-100 and $0.5 \%$ BSA). The secondary antibodies, conjugated with either FITC or rhodamine, were purchased from Santa Cruz Biotechnology Inc. (California, USA). Finally, the egg chambers were mounted in antifading mounting medium and viewed using a Nikon EZ 2000 confocal laser scanning microscope, or a TE 2000-S inverted fluorescent microscope. Routine procedures applied as controls to demonstrate the specificity of the immunostaining protocols used were: (i) the usage of the respective pre-immune serum instead of the antibody serum and (ii) the omission of the respective primary antibody. In all cases, no immunoreactivity could ever be observed.

\section{Monodansylcadaverine staining for autophagic vacuoles}

Monodansylcadaverine (MDC) staining was performed as following: dissected ovaries were briefly rinsed with PBS and immediately transferred into a PBS solution containing 0.05 mM MDC (Fluka, SigmaAldrich, Germany) for 10 min at $25^{\circ} \mathrm{C}$ in the dark (Biederbick et al., 1995; Nezis etal., 2006b). The treated ovaries were washed four times with PBS and immediately observed under a Nikon Eclipse TE 2000-S fluorescent microscope using the appropriate filters.

\section{Transmission electron microscopy}

C. capitata egg chambers were processed for conventional transmission electron microscopy as following: ovaries were dissected in Ringer's solution and fixed in a PBS solution containing $1 \%$ glutaraldehyde and $1 \%$ $\mathrm{OsO}_{4}, \mathrm{pH} 6.2$, for $60 \mathrm{~min}$ at $4^{\circ} \mathrm{C}$. After three washes in distilled water for $5 \mathrm{~min}$ each at $4^{\circ} \mathrm{C}$, the ovaries were stained en bloc overnight in $0.5 \%$ uranyl acetate at $4^{\circ} \mathrm{C}$ in the dark. The specimens were then dehydrated through a graded series of ethanol concentrations, infiltrated in propylene oxide and embedded in Epon-Araldite (Fullam Inc., New York, USA). Ultrathin sections were mounted on uncoated copper grids, stained with uranyl acetate and lead citrate and subsequently observed using a Philips EM 300 electron microscope.

\section{Acknowledgements}

The authors are really grateful to Professors Ron Jemmerson, Claudia Petritsch and Koichi Homma for kindly supplying them with the requested antibodies and to Professor H. M. Moutsopoulos (Department of Pathophysiology, Medical School, University of Athens, Athens, Greece), for kindly providing confocal laser scanning microscope facilities. The authors would also like to thank Ismini Kyriakopoulou and Dr. Ourania Konstandi for their help during the experimental procedures. The present work was co-financed within Op. Education by the ESF (European Social Fund) and National Resources by a grant to Professor L. H. Margaritis (HRAKLEITOS 70/3/7164).

\section{References}

ABRAHAM, E.G., ISLAM, S., SRINIVASAN, P., GHOSH, A.K., VALENZUELA, J.G., RIBEIRO, J.M., KAFATOS, F.C., DIMOPOULOS, G. and JACOBSLORENA, M. (2004). Analysis of the Plasmodiumand Anophelestranscriptional repertoire during ookinete development and midgut invasion. J. Biol. Chem. 279: 5573-5580.

BAUM, J.S., ST. GEORGE, J.P. and MCCALL, K. (2005). Programmed cell death in the germline. Semin. Cell Dev. Biol. 16: 245-259.

BERG, C.A. (2005). The Drosophila shell game: patterning and morphological change. Trends Genet. 21: 346-355.

BIEDERBICK, A., KERN, H.F. and ELSASSER, H.P. (1995). Monodansylcadaverine (MDC) is a specific in vivo marker for autophagic vacuoles. Eur. J. Cel/ Biol. 66: 3-14. 
BURSCH, W., HOCHEGGER, K., TOROK, L., MARIAN, B., ELLINGER, A. and HERMANN, R.S. (2000). Autophagic and apoptotic types of programmed cell death exhibit different fates of cytoskeletal filaments. J. Cell Sci. 113:11891198.

BURSCH, W. (2001). The autophagosomal-lysosomal compartment in programmed cell death. Cell Death Differ. 8: 569-581.

BURSCH, W. (2004). Multiple cell death programs: Charon's lifts to Hades. FEMS Yeast Res. 5: 101-110.

BUSZCZAK, M., FREEMAN, M.R., CARLSON, J.R., BENDER, M., COOLEY, L. and SEGRAVES, W.A. (1999). Ecdysone response genes govern egg chamber development during mid-oogenesis in Drosophila. Development 126: 45814589.

CAVALIERE, V., TADDEI, C. and GARGIULO, G. (1998). Apoptosis of nurse cells at the late stages of oogenesis of Drosophila melanogaster. Dev. Genes Evol. 208: 106-112.

CHAO, S. and NAGOSHI, R.N. (1999). Induction of apoptosis in the germline and follicle layer of Drosophila egg chambers. Mech. Dev. 88: 159-172.

CHWIERALSKI, C.E., WELTE, T. and BUHLING, F. (2006). Cathepsin-regulated apoptosis. Apoptosis 2: 143-149.

CLARKE, P.G. (1990). Developmental cell death: morphological diversity and multiple mechanisms. Anat. Embryol. (Berlin) 181: 195-213.

DANIAL, N.N. and KORSMEYER, S.J. (2004). Cell death: critical control points. Cel/116: 205-219.

DE LORENZO, C., STRAND, D. and MECHLER, B.M. (1999). Requirement of Drosophilal(2)gl function for survival of the germline cells and organization of the follicle cells in a columnar epithelium during oogenesis. Int. J. Dev. Biol. 43: 207-217.

DRUMMOND-BARBOSA, D. and SPRADLING, A.C. (2001). Stem cells and their progeny respond to nutritional changes during Drosophilaoogenesis. Dev. Biol. 231: $265-278$.

FOLEY, K. and COOLEY, L. (1998). Apoptosis in late stage Drosophila nurse cells does not require genes within the $\mathrm{H} 99$ deficiency. Development 125: 10751082.

GIORGI, F. and DERI, P. (1976). Cell death in ovarian chambers of Drosophila melanogaster. J. Embryol. Exp. Morphol. 35: 521-533.

GOZUACIK, D. and KIMCHI, A. (2004). Autophagy as a cell death and tumor suppressor mechanism. Oncogene 23: 2891-2906.

JUHASZ, G. and SASS, M. (2005). Hid can induce, but is not required for autophagy in polyploid larval Drosophila tissues. Eur. J. Cell Biol. 84: 491-502.

KING, R.C. (1970). Origin and development of the egg chamber within the adult ovarioles. In: Ovarian Development in Drosophila melanogaster. Academic Press, New York / London, pp 38-54.

KORNBLUTH, S. and WHITE, K. (2005). Apoptosis in Drosophila: neither fish nor fowl (nor man, nor worm). J. Cell Sci. 118: 1779-1787.

KRIESER, R.J. and WHITE, K. (2002). Engulfment mechanism of apoptotic cells. Curr. Opin. Cell Biol. 14: 734-738.

LAUNDRIE, B., PETERSON, J.S., BAUM, J.S., CHANG, J.C., FILEPPO, D., THOMPSON, S.R. and MCCALL, K. (2003). Germline cell death is inhibited by P-element insertions disrupting the $d c p-1 /$ pita nested gene pair in Drosophila. Genetics 165: 1881-1888.

LOCKSHIN, R.A. and ZAKERI, Z. (2004). Apoptosis, autophagy and more. Int. J. Biochem. Cell Biol. 36: 2405-2419.

MAHAJAN-MIKLOS, S. and COOLEY, L. (1994). Intercellular cytoplasm transport during Drosophila oogenesis. Dev. Biol. 165: 336-351.

MARGARITIS, L.H. (1985). Structure and physiology of the eggshell. In: Gilbert, L.I., Kerkut, G.A. (Eds.), Comprehensive Insect Biochemistry, Physiology and Pharmacology. Pergammon Press, Oxford and New York, Vol. 1, pp 151-230.

MARGARITIS, L.H. (1986). The eggshell of Drosophila melanogaster. New staging characteristics and fine structural analysis of choriogenesis. Can. J. Zool. 64: 2152-2175.

MARTIN, D.N. and BAEHRECKE, E.H. (2004). Caspases function in autophagic programmed cell death in Drosophila. Development 131: 275-284.

MCCALL, K. (2004). Eggs over easy: cell death in the Drosophila ovary. Dev. Biol. 274: 3-14.
MOUZAKI, D.G and MARGARITIS, L.H. (1991). Choriogenesis in the medfly Ceratitis capitata (Wiedermann) (Diptera: Tephritidae). Int. J. Insect Morphol. Embryol. 20: 51-68.

MUELLER, C.M. and JEMMERSON, R. (1996). Maturation of the antibody response to the major epitope on the self antigen mouse cytochrome c. Restricted $\mathrm{V}$ gene usage, selected mutations and increased affinity. J. Immunol. 157: 5329-5338.

MULLER, F., ADORI, C. and SASS, M. (2004). Autophagic and apoptotic features during programmed cell death in the fat body of the tobacco hornworm (Manduca sexta). Eur. J. Cell Biol. 83: 67-78.

NEZIS, I.P., STRAVOPODIS, D.J., PAPASSIDERI, I., ROBERT-NICOUD, M. and MARGARITIS, L.H. (2000). Stage-specific apoptotic patterns during Drosophila oogenesis. Eur. J. Cell Biol. 79: 610-620.

NEZIS, I.P., STRAVOPODIS, D.J., PAPASSIDERI, I. and MARGARITIS, L.H. (2001). Actin cytoskeleton reorganization of the apoptotic nurse cells during the late developmental stages of oogenesis in Dacus oleae. Cell Motil. Cytoske/eton 48: 224-233.

NEZIS, I.P., STRAVOPODIS, D.J., PAPASSIDERI, I., ROBERT-NICOUD, M. and MARGARITIS, L.H. (2002). The dynamics of apoptosis in the ovarian follicle cells during the late stages of Drosophila oogenesis. Cell Tissue Res. 307: 401409.

NEZIS, I.P., MODES, V., MPAKOU, V., STRAVOPODIS, D.J., PAPASSIDERI, I.S., MAMMALI, I. and MARGARITIS, L.H. (2003). Modes of programmed cell death during Ceratitis capitata oogenesis. Tissue Cel/35: 113-119.

NEZIS, I.P., STRAVOPODIS, D.J., PAPASSIDERI, I.S., STERGIOPOULOS, C. and MARGARITIS, L.H. (2005). Morphological irregularities and features of resistance to apoptosis in the $d c p-1 /$ pita double mutated egg chambers during Drosophila oogenesis. Cell Motil. Cytoskeleton 60: 14-23.

NEZIS, I.P., STRAVOPODIS, D.J., MARGARITIS, L.H. and PAPASSIDERI, I.S. (2006a). Follicular atresia during Dacus oleae oogenesis. J. Insect Physiol. 52 : 282-290.

NEZIS, I.P., STRAVOPODIS, D.J., MARGARITIS, L.H. and PAPASSIDERI, I.S. (2006b). Programmed cell death of follicular epithelium during the late developmental stages of oogenesis in the fruit flies Bactrocera oleae and Ceratitis capitata (Diptera, Tephritidae) is mediated by autophagy. Dev. Growth Differ. 48: 189-198.

NEZIS, I.P., STRAVOPODIS, D.J., MARGARITIS, L.H. and PAPASSIDERI, I.S. (2006c). Chromatin condensation of ovarian nurse and follicle cells is regulated independently from DNA fragmentation during Drosophila late oogenesis. Differentiation 74: 293-304.

PETERSON, J.S., BARKETT, M. and MCCALL, K. (2003). Stage-specific regulation of caspase activity in Drosophila oogenesis. Dev. Biol. 260: 113-123.

RAIKHEL, A.S., KOKOZA, V.A., ZHU, J., MARTIN, D., WANG, S.F., LI, C., SUN, G., AHMED, A., DITTMER, N. and ATTARDO, G. (2002). Molecular biology of mosquito vitellogenesis: from basic studies to genetic engineering of antipathogen immunity. Insect Biochem. Mol. Biol. 32: 1275-1286 (Review).

ROSSITER, J.P. ANDERSON, L.L., YANG, F. and COLE, G.M. (2000). Caspasecleaved actin (fractin) immunolabelling of Hirano bodies. Neuropathol. Appl. Neurobiol. 4: 342-346.

SOLLER, M., BOWNES, M. and KUBLI, E. (1999). Control of oocyte maturation in sexually mature Drosophila females. Dev. Biol. 208: 337-351.

TERASHIMA, J. and BOWNES, M. (2005). A microarray analysis of genes involved in relating egg production to nutritional intake in Drosophila melanogaster. Cell Death Differ. 12: 429-440.

TROUGAKOS, I.P. and MARGARITIS, L.H. (2002). Novel morphological and physiological aspects of insect eggs. In: Hilker, M., Meiners, T. (Eds.), Chemoecology of Insect Eggs and Egg Deposition, Blackwell Wissenschaftsverlag, Berlin, Germany, pp 3-36.

UCHIDA, K., OHMORI, D., UENO, T., NISHIZUKA, M., ESHITA, Y., FUKUNAGA, A. and KOMINAMI, E. (2001). Preoviposition activation of cathepsin-like proteinases in degenerating ovarian follicles of the mosquito Culexpipiens pallens. Dev. Biol. 237: 68-78.

UCHIDA, K., NISHIZUKA, M., OHMORI, D., UENO, T., ESHITA, Y. and FUKUNAGA, A. (2004). Follicular epithelial cell apoptosis of atretic follicles within developing ovaries of the mosquito Culex pipiens pallens. J. Insect Physiol. 50: 903-912. VARKEY, J., CHEN, P., JEMMERSON, R. and ABRAMS, J.M. (1999). Altered 
66 A.D. Velentzas et al.

cytochrome c display precedes apoptotic cell death in Drosophila. J. Cell Biol. 144: 701-710.

VERRAS, M., GOURZI, P., ZACHAROPOULOU, A. and MINTZAS, A.C. (2002). Developmental profiles and ecdysone regulation of the mRNAs for two ecdysone receptor isoforms in the Mediterranean fruit fly Ceratitis capitata. Insect Mol. Biol. 11: 553-565.

WALDHUBER, M., EMOTO, K. and PETRITSCH, C. (2005). The Drosophila caspase DRONC is required for metamorphosis and cell death in response to irradiation and developmental signals. Mech. Dev. 122: 914-927.
YANG, F., SUN, X., BEECH, W., TETER, B., WU, S., SIGEL, J., VINTERS, H.V., FRAUTSCHY, S.A. and COLE, G.M. (1998). Antibody to caspase-cleaved actin detects apoptosis in differentiated neuroblastoma and plaque-associated neurons and microglia in Alzheimer's disease. Am. J. Pathol. 152: 379-389.

Received: 20th March 2006 Reviewed by Referees: 15th May 2006 Modified by Authors and Accepted for Publication: 3rd July 2006 Published Online: 5th October 2006 